\title{
Magnetic Resonance Enterography in 300 Known Cases of Crohn's Disease: An Initial Report from a Referral Center in Iran
}

\author{
Amir Reza Radmard ${ }^{1 *}$, Sepehr Haghighi ${ }^{1}$, Amir Pejman Hashemi Taheri ${ }^{1}$, Payam Mohammadinejad ${ }^{1}$, \\ Rana Eftekhar Vaghefi ${ }^{1}$, Sara Naybandi Atashi ${ }^{1}$, Neda Pak ${ }^{1}$, Leila Aghaghazvini ${ }^{1}$, Reza Malekzadeh ${ }^{2}$
}

1. Department of Radiology, Shariati hospital, Tehran University of Medical Sciences, Tehran, Iran.

2. Autoimmune and Motility Disorders of the Gastro-Intestinal Tract Research Center, Digestive Diseases Research Institute, Tehran University of Medical Sciences, Tehran, Iran.

\section{* Corresponding Author:}

Amir Reza Radmard, MD Assistant professor, Department of Radiology, Tehran University of Medical Sciences, Shariati Hospital, 14117, North Kargar St.,Tehran, Iran.

Tel: + 982182415300

Fax: + 982182415400

Email:Radmard@ams.ac.ir

Received: 19 Apr. 2016

Accepted: 18 Aug. 2016

\section{ABSTRACT}

\section{BACKGROUND}

Magnetic resonance enterography (MRE) has become the modality of choice in assessment of patients with Crohn's disease (CD). We aimed to present our experience on 300 patients with CD who underwent MRE during the first 30 months after setting up MRE for the first time in a referral center in Iran.

\section{METHODS}

Patients with a definite diagnosis of CD based on either ileocolonoscopy or histopathological studies were included in the final report and categorized into four phenotypes of inactive, active, stricturing, and penetrating disease.

\section{RESULTS}

This was a case series study on 300 patients with known CD out of 594 referred subjects. The most prevalent phenotype was inactive observed in 162 $(54.0 \%)$ patients followed by stricturing in $44(14.7 \%)$, active in $40(13.3 \%)$, penetrating in $27(9 \%)$, and active on chronic in $27(9 \%)$ cases. The number of referred patients increased from 51 cases in the first 6 months to 165 in the last 6 months.

\section{CONCLUSION}

This study presents the first report on the application of MRE in Iran as superb modality for management of $\mathrm{CD}$. The growing number of referred patients indicates that MRE has been successful in addressing the most critical concerns of clinicians on determining the dominant disease phenotype.

KEYWORDS:Crohn's Disease; Magnetic Resonance Enterography; Diagnosis; Iran

Please cite this paper as:

Radmard AR, Haghighi S, Hashemi Taheri AP, Mohammadinejad P, Eftekhar Vaghefi R, Naybandi Atashi S, Pak N, Aghaghazvini L, Malekzadeh R. Magnetic Resonance Enterography in 300 Known Cases of Crohn's Disease: An Initial Report from a Referral Center in Iran. Middle East J Dig Dis 2016;8:273-281. DOI: 10.15171/mejdd.2016.36

\section{INTRODUCTION}

Crohn's disease (CD) is a chronic inflammatory disease involving the gastrointestinal tract with an unpredictable course. The peak age of the onset of $\mathrm{CD}$ is in the second to fourth decades of life, and its course follows a pattern of periodic recurrences and exacerbations. ${ }^{1} \mathrm{CD}$ is a multiorgan disease, which mainly involves the small bowel and colon, but other parts of the gastrointestinal tract may also be affected. CD manifests with bowel wall inflammation, erosions, ulcerations, and non-caseating granulomas. Bowel is frequently affected segmentally, with healthy areas between the involved segments called 
"skip lesions". The mucosa is affected first, forming aphthoid ulceration as well as lymphoid hyperplasia, and then followed by longitudinal and horizontal ulcers. Transmural ulcer formation is the next complication, forming sinuses, fistulas, and abscesses. In chronic CD, bowel inflammation results in fatty infiltration involving the lumen wall, accompanied by fibrofatty proliferation observed in the nearby mesenteric fat. Moreover, stricture and the subsequent obstruction of the bowel lumen could be resulted from the fibrosis. In some cases, signs of acute and chronic CD may concur in the same bowel segment. Patients with $C D$ usually undergo numerous imaging investigations either before the diagnosis or for follow-up evaluations, exposing them to ionizing radiation, often beginning in the adolescence. ${ }^{1}$

In the evaluation of $\mathrm{CD}$, multiple diagnostic imaging methods have been developed. There is still a need for complementary study and conclusive validation of the optimal deployment of the currently available imaging modalities in combination with endoscopic and histopathological studies. Numerous authors have recommended the use of computed tomography (CT) in the evaluation of patients suspected with $\mathrm{CD}$ because of its accuracy and feasibility. ${ }^{2,3}$ On the other hand, abdominal CT exposes the patients to a significant amount of radiation, putting these individuals at a greater risk for cancers. ${ }^{4}$

Magnetic resonance imaging (MRI), on the other hand, is a non-invasive imaging modality without ionizing radiation. There has been an increasing trend in the use of this modality for the evaluation of gastrointestinal tract and its nearby organs in patients with suspected or confirmed diagnosis of CD. ${ }^{5,6}$ Since MRI is less sensitive compared with colonoscopy in the evaluation of mucosa, there may be high rates of false-negative results in MRI studies, especially in CD cases with mild or superficial bowel involvement. ${ }^{?}$

The degree of disease activity is hard to determine in patients with $\mathrm{CD}$ and it frequently requires the combined use of clinical, endoscopic, and imaging studies. ${ }^{8}$ Furthermore, routine modalities such as conventional barium studies offer little insight regarding the disease activity and the extramural involvement, most notably, abscess formation.

Magnetic Resonance Enterography (MRE) provides precise evaluation of the extraluminal complications of
$\mathrm{CD}$, its distribution and the level of activity, and the evaluation of bowel segments proximal to bowel strictures, which are inaccessible via colonoscopy. ${ }^{2,9}$ This characteristic, along with non-invasiveness and non-ionizing radiation features of the MRE has made it a modality of choice particularly in the long-term follow-up of patients with $\mathrm{CD}$.

In this manuscript, we present our experience with MRE in a large number of patients with proven diagnosis of $\mathrm{CD}$, for the first time in Iran, who visited our referral center over a 30-month period, to present the spectrum of imaging findings and discuss different $\mathrm{CD}$ phenotypes. We also aimed to describe our experience about the technical aspects of MRE and describe the necessary information for radiologists to perform and appreciate MRE.

\section{MATERIALS AND METHODS}

The current study was approved by our hospital Institutional Review Board and informed consent was obtained from all patients before data entry.

\section{Study Subjects}

This is a case series study on 300 patients with known CD out of 594 subjects who were referred to Shariati Hospital affiliated to Tehran University of Medical Sciences between October 2012 and March 2014 for evaluation of small bowel by MRE. Definite diagnosis of CD was made based on either ileocolonoscopy or histopathological results. Accordingly the excluded 294 cases out of the 594 subjects consisted of those suspected for CD without definite diagnosis and those cases with small bowel abnormalities other than CD.

All included subjects aged 15 years and more. Exclusion criteria were pregnancy, diabetes, and known renal dysfunction. Demographic information and written consent were obtained from all the patients. All MR images were analyzed by a single board certified radiologist with more than 4 years' experience in abdominal imaging.

\section{Oral preparation}

The patients were asked to have a low-residue diet for the preceding $48 \mathrm{~h}$ and fast for at least $6 \mathrm{~h}$ prior to MRI. To achieve sufficient distension of small bowel, all subjects drank $1500 \mathrm{~mL}$ polyethylene glycol (PEG) solution including three aliquots over $45 \mathrm{~min}$ before MRE. Each 
aliquot was drunk over $15 \mathrm{~min}$, which consisted of 2 packets ( $25 \mathrm{gr}$ ) of PEG dissolved in $500 \mathrm{~mL}$ of tap water. In case of inadequate small bowel distension, the patients were asked to drink additional $500 \mathrm{~mL}$ water during the next $15 \mathrm{~min}$. A metoclopramide tablet $(10 \mathrm{mg})$ was given with the first aliquot to accelerate gastric emptying. 20 mg hyoscine N-butyl bromide was administered intravenously to decrease bowel movements when satisfactory distension could be achieved following the first obtained sequence (Coronal T2-HASTE).

The MRE examinations were categorized based on the quality of small bowel distension as satisfactory and unsatisfactory. Satisfactory imaging was a subjective term defined by the radiologist to describe the quality of the imaging study, which addressed whether the adequate distension of small bowel had been achieved. This is a routine technical point in MRE examinations guiding clinicians about the sensitivity of the exam, which might be affected by inadequate distension of small bowel described as unsatisfactory. In such cases a repeated exam or short term follow-up MRE might be recommended by radiologists especially when the findings are not correlated well with the clinical setting. However it should be noted that in case of no small bowel distension, which might lead to a totally non-diagnostic exam, the procedure would be canceled and they were not included in this study.

\section{Imaging technique}

All MREs were performed using a 1.5-T MR scanner (Avanto; Siemens, Erlangen, Germany) having an 18-channel torso-phased-array coil long enough to cover the entire small bowel. The MRE protocol included coronal, sagittal, and axial T2-weighted half-Fourier singleshot turbo spin-echo (HASTE) $(\mathrm{TR}=1500 \mathrm{~ms}$; TE=92 $\mathrm{ms}$; echo train length $=227$; section thickness $=5 \mathrm{~mm}$; no gap; FOV=400-450 mm; matrix $=227 \times 384$ ), coronal true fast imaging in steady-state precession (True-FISP) $(\mathrm{TR}=3.9 \mathrm{~ms} ; \mathrm{TE}=2 \mathrm{~ms}$; flip angle 60 ; section thickness $=5 \mathrm{~mm}$; no gap; FOV=350 mm; matrix $=230 \times 256$ ) and coronal HASTIRM $(\mathrm{TR}=1350 \mathrm{~ms}$; $\mathrm{TE}=82 \mathrm{~ms}$; flip angle 150; section thickness $=5 \mathrm{~mm}$; no gap; $\mathrm{FOV}=450$ $\mathrm{mm}$; matrix $=248 \times 384$ ) sequences. Before injecting intravenous contrast material, coronal volumetric interpolated breath-hold examination (VIBE) $(\mathrm{TR}=4.8 \mathrm{~ms}$;
$\mathrm{TE}=2.3 \mathrm{~ms}$; $1 \mathrm{slab}$, slice per slab: 72; flip angle 10 ; section thickness $=3 \mathrm{~mm}$; gap $=0.6 \mathrm{~mm}$; FOV $=480 \mathrm{~mm}$; matrix $=177 \times 320$ ) images were obtained through the abdomen and pelvis. Three additional coronal VIBE images were acquired at 30,70, and 180 seconds after injection of $10 \mathrm{~mL}$ gadopentetate dimeglumine (Magnevist; Berlex Laboratories, Montville, NJ).

Finally, the small intestine of the patients was assessed based on mural thickening, number, location, and length of involved segments, luminal narrowing, stricture, enhancement pattern, mesenteric vascularity, mesenteric lymphadenopathy, fistula, and presence of collection. The imaging phenotypes of $\mathrm{CD}$ were also determined in all the patients, including quiescent (inactive), active, stricturing, penetrating, and active on chronic types.

\section{Definitions}

Mural thickening was defined as the mural thickness of more than $3 \mathrm{~mm}$ in a well distended small bowel loop. Stricture was determined when luminal narrowing was accompanied by proximal bowel dilation more than 3 $\mathrm{cm}$ in luminal diameter. Active $\mathrm{CD}$ was presumed as the presence of mural thickening, T2 hyper intensity of bowel wall, increased contrast enhancement and mesenteric engorgement in jejunal or ileal loops. Inactive (quiescent) CD was defined as segmental mural thickening with mildly increased contrast enhancement or without abnormal enhancement, which was not accompanied by mesenteric engorgement, stricture, fistula or abscess. Chronic CD was determined as mural thickening in small bowel with mildly increased contrast enhancement or normal enhancement without mesenteric engorgement, which was categorized into stricturing and penetrating (fistulizing) disease based on the presence of a stricture or fistula/abscess, respectively.

\section{Statistical Analysis}

All data were analyzed using SPSS software for windows version 17 (SPSS Corp, USA). Quantitative data are presented as mean \pm standard deviation (SD) and qualitative data as number (percentage). Chi-square test was deployed to compare different features between the groups. Differences were considered as statistically significant if $\mathrm{p}$ value $<0.05$. 


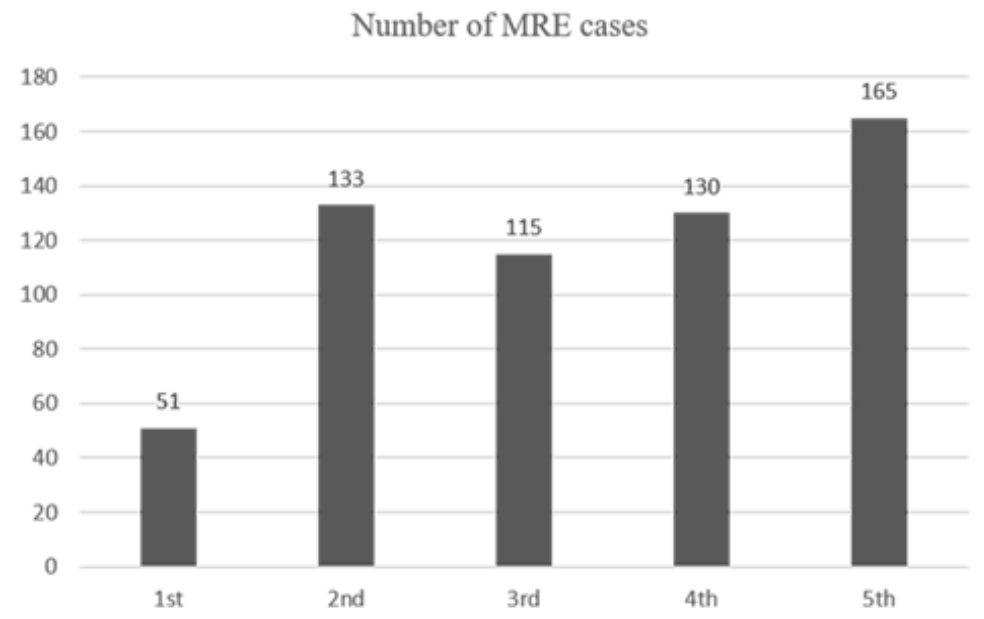

Fig.1: Number of cases referred for magnetic resonance enterography in 6-month intervals.

\section{RESULTS}

A total of 594 patients were evaluated during the study period. Of them, 300 patients (50.5\%) met the definite diagnosis of $\mathrm{CD}$ based on either ileocolonoscopy or histopathological studies. There was an increasing trend in the number of cases referred to our center for MRE except for the 2nd and 3rd 6-month intervals in the 2.5year period of the study. Figure 1 shows the distribution of referred patients in 6-month intervals.

A total of 300 patients with $\mathrm{CD}$, including 160 women $(53.3 \%)$ and 140 men $(46.7 \%)$ were included in the final report. The mean age of the patients was $37.42 \pm 14.41$ years (range: 15-77 years) with a median of 36 years. MRE technique was considered as unsatisfactory in about one-third of the cases. The quality of MRE technique was not influenced by the operator experience. Of the 300 patients, 198 (66\%) had abnormal MRE findings related to $\mathrm{CD}$ and all of these cases had signs of mural thickening. Single segment involvement was observed in MRE in 82 (27.3\%), 2 or 3 segments in 40 (13.3\%), and more than 3 segments in $76(25.3 \%)$ patients. In the remaining 102 patients (34.0\%) with unremarkable MRE, there was no $\mathrm{CD}$ related small bowel abnormality. The lengths of the involved segments were $<5 \mathrm{~cm}$ in 79 cases $(26.3 \%), \geq 5 \mathrm{~cm}$ in 81 cases $(27 \%)$, and with at least one segment $<5 \mathrm{~cm}$ and one segment $\geq 5 \mathrm{~cm}$ in 38 cases $(12.7 \%)$.

Ileum was the most common site of involvement observed in 179 cases equal to $59.7 \%$ of the total cases or
$90.4 \%$ of cases with abnormal MRE. Out of the 300 cases, concurrent colon and ileum involvements were found in $36(12 \%)$ cases, while coexistence of ileal and jejunal involvement was observed in $9(3 \%)$ cases. In 19 (6.3\%) cases, single involvement of the colon was detected. Luminal narrowing and luminal stricture were observed in $141(47 \%)$ and $56(18.7 \%)$ cases, respectively. Increased contrast enhancement was visualized in 100 (33.3\%) patients while intensely increased enhancement was delineated in $80(26.7 \%)$ cases. Increased mesenteric vascularity and the presence of mesenteric lymphadenopathy were found in 120 cases $(40 \%)$ and 159 cases $(53 \%)$, respectively. Fistula was present in MRE of 37 (12.3\%) cases described in detail in table 1.

The most prevalent phenotype of $\mathrm{CD}$, as evaluated by MRE, was inactive type observed in 162 cases $(54.0 \%)$. This was followed by stricturing in 44 (14.7\%), active in $40(13.3 \%)$, penetrating in $27(9 \%)$, and active on chronic in $27(9 \%)$ patients.

We also compared MRE findings in patients with chronic $\mathrm{CD}$ versus those with active on chronic $\mathrm{CD}$. Compared with $\mathrm{CD}$ patients with chronic disease, those with active on chronic disease had significantly higher rates of intensely increased enhancement patterns (35.2\% vs. $96.3 \%, p<0.001)$, luminal narrowing without proximal dilation $(85.9 \%$ vs. $100 \%, \mathrm{p}=0.033)$, luminal stricture $(40.8 \%$ vs. $96.3 \%, \mathrm{p}<0.001)$. Other MRE findings were not significantly different between these two groups of patients. Table 2 compares MRE findings 
Table 1: Types of fistula in magnetic resonance enterography of the patients with CD

\begin{tabular}{lc}
\hline Types of fistula & No (\%) \\
\hline Perianal & $17(5.7 \%)$ \\
\hline Enteroenteric & $7(2.3 \%)$ \\
\hline Enterocolic & $3(1 \%)$ \\
\hline Enterocutaneous & $6(2 \%)$ \\
\hline Enterovesical & $1(0.3 \%)$ \\
\hline Rectovaginal & $1(0.3 \%)$ \\
\hline Combined & $2(0.7 \%)$ \\
\hline Total No & $37(12.3 \%)$ \\
\hline
\end{tabular}

in patients with chronic $\mathrm{CD}$ versus those with active on chronic CD. Few samples of different $\mathrm{CD}$ phenotypes are shown in figures 2, 3, 4 and 5

\section{DISCUSSION}

According to our findings, about half of the patients who underwent MRE for probable CD were diagnosed as having this disorder based on ileocolonoscopy or histopathological studies. Moreover, we had about 20 new referred cases each month after 6 months of the setting up of MRE as a new imaging modality in our center. This trend could be attributed to the satisfaction of clinicians from MRE modality in providing straightforward answers to their questions about the management of patients with $\mathrm{CD}$ by determining the dominant phenotype of the disease. Each phenotype of $\mathrm{CD}$ requires a different approach for the management or treatment in the clinical setting. The growing number of the referred patients over the 30-month period indicates that the application of MRE has been successful in addressing the most critical concerns of physicians in the diagnosis, follow-up, and treatment of patients with CD. Despite the wide acknowledgement of MRE in the diagnosis of CD in industrialized countries, its availability and application in Iran is not well known to the most of the country's radiologists and gastroenterologists. Our experience shows that upon the availability of this modality, clinicians are eager to use it in their daily practice. This is of importance since the prevalence of CD in Iran has supposedly increased in recent years. ${ }^{10}$ Eventhough, the exact prevalence of CD in Iran is not precisely known, apparently there has been an increasing trend in the observation of new cases as a result of the steady adoption of a lifestyle more resembling to the western countries. ${ }^{10}$

Assessment of the small intestine via imaging studies has remained a challenging issue. A perfect diagnostic modality should be non-invasive, without ionizing radiation that can clearly display the bowel lumen as well as extraintestinal tissues. CD is a chronic and incurable disease, commonly observed in young individuals. ${ }^{11}$ Patients with CD should be monitored regularly since early detection of complications could prevent the need for more invasive therapeutic interventions such as the surgical resection of parts of the intestine.

Imaging modalities are non-invasive and could detect extraluminal pathologies. Moreover, in contrast to endoscopic and colonoscopic modalities, which cannot pass the strictures caused by $\mathrm{CD}$, they can easily show the whole gastrointestinal tract. Among these modalities, the use of ultrasonography is limited by the operator's experience, and is not able to detect many of the complications of CD. ${ }^{12}$ Computed tomography enterography (CTE) have the advantages of high spatial resolution and short acquisition time, and the ability to determine the activity of disease, enhancement of the bowel wall, and extraluminal complications. ${ }^{13,14}$ However, CTE exposes the patients to high amounts of ionizing radiation. Since such patients should be evaluated regularly for decades, the effects of cumulative doses of ionizing radiation create genuine concerns. ${ }^{15}$ There has been an ever increasing interest in MRE as a substitute for standard modalities both in the diagnosis and follow-up of patients with $\mathrm{CD}$. Some authors even recommend MRE as a first line modality in the diagnosis of $\mathrm{CD}$ given to its safety and acceptable accuracy. ${ }^{16}$ MRE is considered as a first choice modality in the detection of perianal fistula, which is known to be more accurate than CTE, endosonography, and physical examination. ${ }^{17,18}$ Moreover, MRI is more accurate in the assessment of soft tissue, compared with CTE, even if performed without the administration of contrast material. Another advantage of MRI over CT is its applicability in pregnant patients and in patients with impaired renal function. ${ }^{19,20}$

MRE is not only an excellent modality to diagnose $\mathrm{CD}$, but can provide superior diagnostic information regarding to the differentiation of various phenotypes of the disease, including quiescent (inactive), active, stricturing, or penetrating disease. The degree of dis- 
Table 2: Comparison between patients with chronic and active on chronic Crohn's disease

\begin{tabular}{|c|c|c|c|}
\hline Variables & $\begin{array}{l}\text { Active on chronic } \\
\quad(\mathrm{n}=27)\end{array}$ & $\begin{array}{c}\text { Chronic (stricturing+ fistulizing) } \\
\qquad(\mathrm{n}=71)\end{array}$ & P value \\
\hline Enhancement Pattern & & & $<0.001$ \\
\hline Normal & 0 & $6(8.5 \%)$ & \\
\hline Increased & $1(3.7 \%)$ & $40(56.3 \%)$ & \\
\hline Intense increased & $26(96.3 \%)$ & $25(35.2 \%)$ & \\
\hline Number of involved segments & & & 0.747 \\
\hline None & 0 & $3(4.2 \%)$ & \\
\hline Single & $9(33.3 \%)$ & $24(33.8 \%)$ & \\
\hline Few ( $\leq 3$ segments) & $6(22.2 \%)$ & $14(19.7 \%)$ & \\
\hline Multiple ( $>3$ segments) & $12(44.4 \%)$ & $30(42.3 \%)$ & \\
\hline Location of involved segments & & & 0.129 \\
\hline None & 0 & $3(4.2 \%)$ & \\
\hline Ileum & $24(88.9 \%)$ & $47(66.2 \%)$ & \\
\hline Colon & 0 & $7(9.9 \%)$ & \\
\hline Ileum and colon & $3(11.1 \%)$ & $9(12.7 \%)$ & \\
\hline Ileum and jejunum & 0 & $5(7 \%)$ & \\
\hline Length of involved segments & & & 0.444 \\
\hline None & 0 & $3(4.2 \%)$ & \\
\hline Short $(<5 \mathrm{~cm})$ & $11(40.7 \%)$ & $19(26.8 \%)$ & \\
\hline Long $(\geq 5 \mathrm{~cm})$ & $10(37 \%)$ & $30(42.3 \%)$ & \\
\hline Short and long & $6(22.2 \%)$ & $19(26.8 \%)$ & \\
\hline Mesenteric lymphadenopathy & & & 0.006 \\
\hline No or non-significant & $2(7.4 \%)$ & $24(33.8 \%)$ & \\
\hline Yes & $25(92.6 \%)$ & $47(66.2 \%)$ & \\
\hline Luminal narrowing & & & 0.033 \\
\hline No & 0 & $10(14.1 \%)$ & \\
\hline Yes & $27(100 \%)$ & $61(85.9 \%)$ & \\
\hline Luminal stricture & & & $0<0.001$ \\
\hline No & $1(3.7 \%)$ & $42(59.2 \%)$ & \\
\hline Yes & $26(96.3 \%)$ & $29(40.8 \%)$ & \\
\hline Fistula & & & 0.107 \\
\hline No & $22(81.5 \%)$ & $47(66.2 \%)$ & \\
\hline Yes & $5(18.5 \%)$ & $24(33.8 \%)$ & \\
\hline Collection & & & 0.177 \\
\hline No & $26(96.3 \%)$ & $62(87.3 \%)$ & \\
\hline Yes & $1(3.7 \%)$ & $9(12.7 \%)$ & \\
\hline
\end{tabular}

ease activity of CD could be assessed by clinical and/ or biochemical data. The Crohn's disease Activity Index (CDAI) is a widely used scoring system applying both these parameters. However it has some limitations such as; (a) subjects with chronic and very mildly active CD or relatively inactive $\mathrm{CD}$ might be over diagnosed as active phenotype, (b) cumbersome scoring system, which needs a long list of symptoms in a week provided by the CD patient, and (c) it could not provide accurate information about sites of disease activity in patients who have $\mathrm{CD}$ with multifocal involvement. ${ }^{21}$ Equal accuracy of CTE and MRE for the recognition of active $\mathrm{CD}$ and its extra-enteric complications has been found. ${ }^{22} \mathrm{~A}$ number of MRE parameters have been linked to active or chron- 


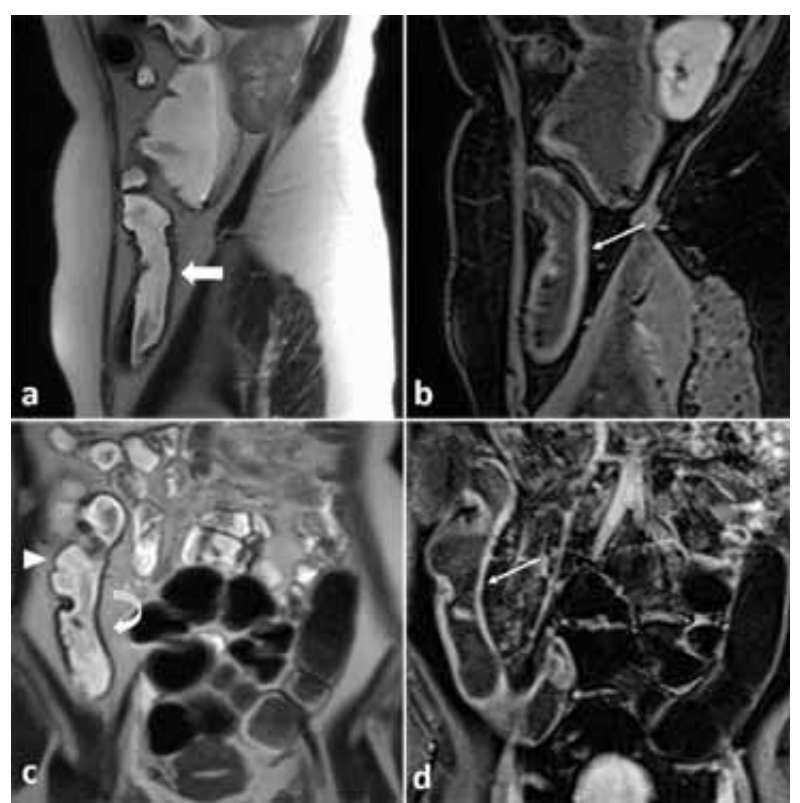

Fig.2: MRE images of a 46-year-old female patient with quiescent Crohn's disease. Sagittal (a) and coronal (c) T2-weighted HASTE (half-Fourier acquisition single-shot turbo spin-echo) sequence show mild mural thickening in terminal ileum (arrow) with dominant mesenteric side involvement (curved arrow) and mild sacculation in antimesenteric side (arrow head). Post contrast sagittal (b) and coronal (d) T1-weighted VIBE (volumetric interpolated breath-hold examination) images depict mild contrast enhancement (thin arrows) in the affected terminal ileal loop.

ic CD in different studies. A recent meta-analysis by Church and colleagues on 62 MRE based studies found 22 signs to represent inflammation and 9 signs to represent chronic damage. The researchers found that wall enhancement, the presence of mucosal lesions, and the observation of wall $\mathrm{T} 2$ hyperintensity were the most useful indicators of inflammation, and the presence of abscess and fistula were the most reliable indicator of damage. ${ }^{23}$

It should be noted that acute inflammation usually coexist with underlying fibrosis and overlap of different $\mathrm{CD}$ phenotypes might be challenging for the radiologist. It is clear that chronic fibrosis can lead to wall thickening; therefore a simple morphological finding like wall thickening as an isolated marker is not accurate enough to predict disease activity. A combination of imaging findings must be considered for detection of active disease. Punwani and co-workers showed that the increase in mural thickness, high values of mural signal intensity on T2-weighted fat-saturated images, and contrast enhancement with a layered pattern reflects acute

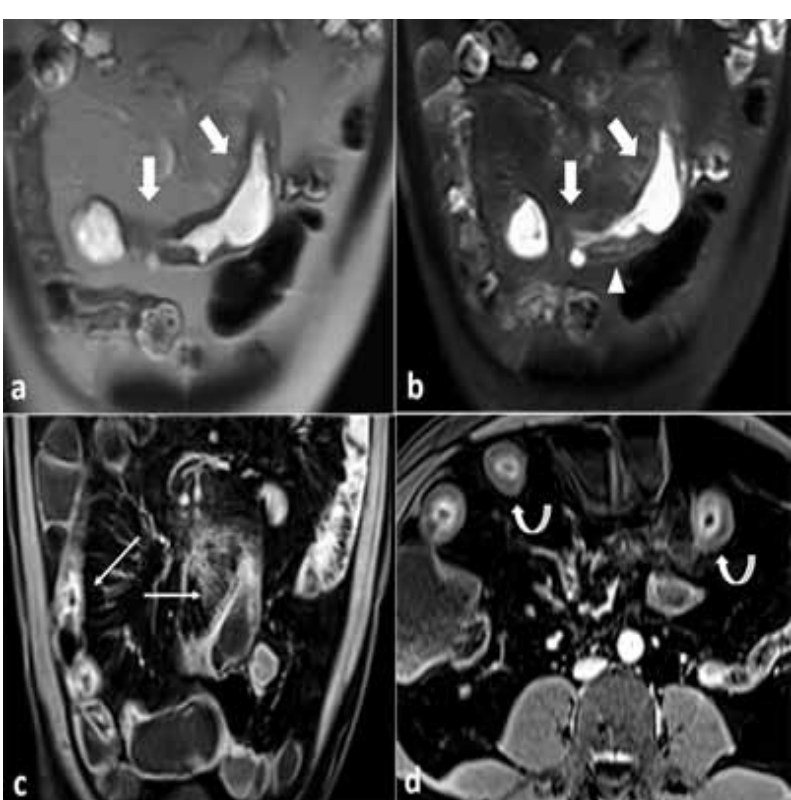

Fig.3: Active Crohn's disease in a 22-year-old male patient. Coronal T2-weighted HASTE (half-Fourier acquisition single-shot turbo spin-echo) (a) and HASTIRM (half-Fourier acquisition singleshot turbo spin-echo using inversion recovery) (b) images show a thickened mid ileal loop with dominant mesenteric side involvement (arrows) and T2-hyperintenstiy of thickened wall (arrow head) due to mural edema. There is intense contrast enhancement in affected ileal loops in coronal (c) and axial (d) post contrast T1weighted images with mesenteric engorgement (thin arrows) and typical target sign (curved arrows).

inflammation in CD by histopathological evaluation of resected segments of small bowel. ${ }^{24}$ In these series, we also applied a constellation of MRE findings, including mural thickening, T2 hyperintensity of bowel wall, increased contrast enhancement (target appearance) and mesenteric engorgement to make a diagnosis of active CD.

The current study is a preliminary investigation and has several limitations. Firstly, it would be ideal to compare the findings of MRE with clinical and laboratory findings of the patients especially scoring systems like CDAI. Secondly, the spectrum of imaging findings of this study should be considered in the setting of a single center and may not completely apply to other populations of patients with CD. Thirdly, it would be ideal to include control subjects who had undergone ileocolonoscopy or histopathological studies for reasons other than $\mathrm{CD}$ in order to investigate the accuracy of MRE in the diagnosis of $\mathrm{CD}$ and its complications.

In conclusion, the current study provides insight into 


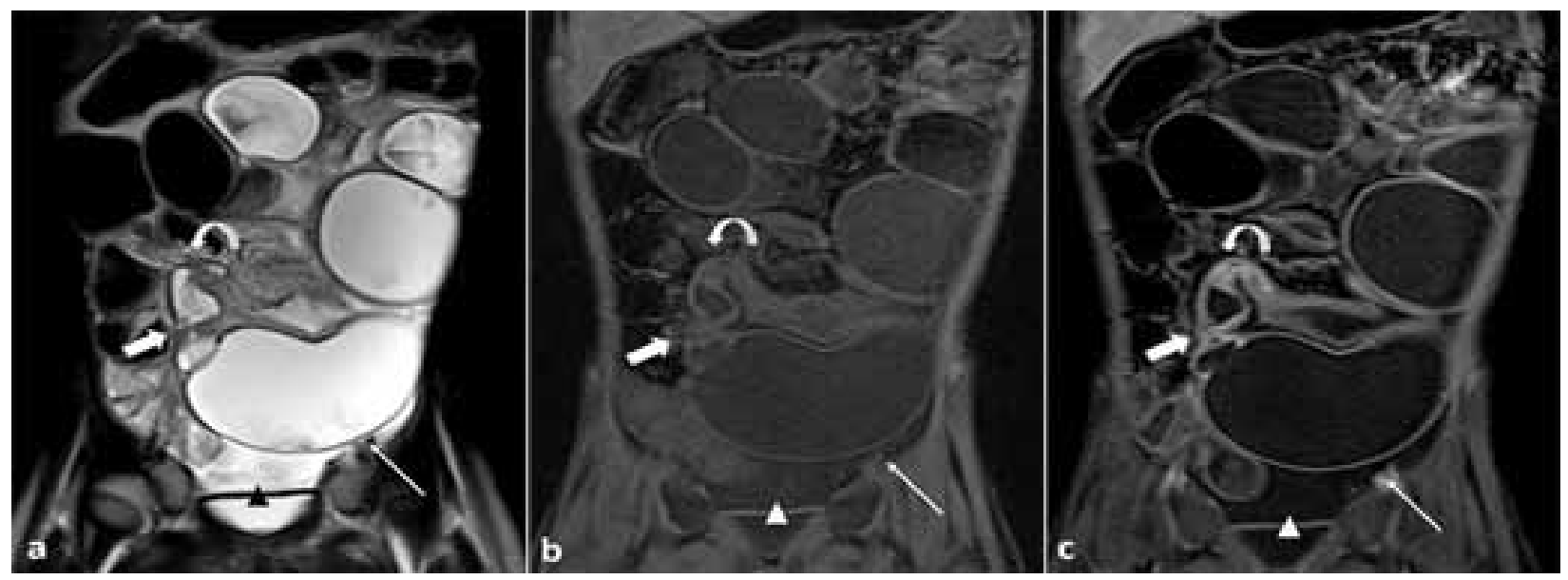

Fig.4: A 38-year-old male patient with Crohn's disease showing stricturing phenotype. Coronal T2-weighted True-FISP (fast imaging with steady-state free precession) image (a) shows two stenotic segments (arrow and curved arrow) in mid ileum with proximal dilation of small bowel (thin arrow). In pre (b) and post (c) contrast coronal T1-weighted images, there is mild enhancement in stenotic ileal segments without adjacent mesenteric engorgement suggestive of chronic disease. Mild free fluid (arrow head) is seen in inter loop space in lower abdomen.
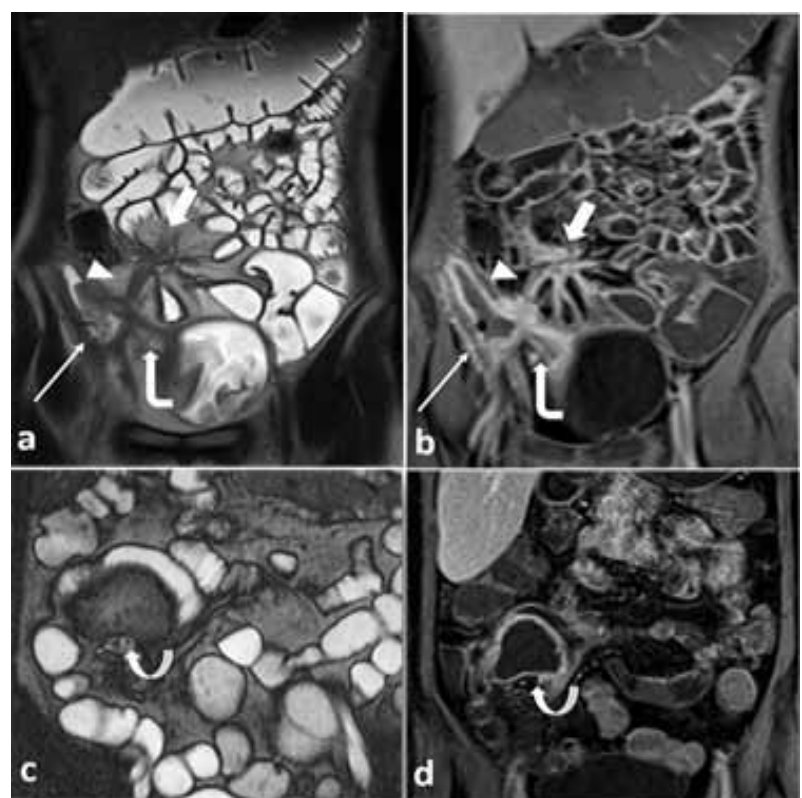

Fig.5: Coronal T2-weighted HASTE (half-Fourier acquisition single-shot turbo spin-echo) (a) and post contrast T1-weighted (b) images of a patient with penetrating Crohn's disease showing convergence of ileal loops in right lower quadrant with ileoileal (arrows) and ileocutaneous (arrow heads) fistulas causing a subcutaneous inguinal abscess (thin arrows). There is also a colocutaneous fistula (bent arrow). Another patient with interloop abscess (curved arrow) adjacent to terminal ileum due to penetrating Crohn's disease in coronal T2-weighted True-FISP (fast imaging with steady-state free precession) (c) image, which shows mural enhancement in post contrast (d) image. the setting up of MRE for the first time in a referral center in Iran as a developing country. MRE may be beneficial in providing guidance for physicians at the clinical setting by categorizing patients based on the subtype of $\mathrm{CD}$. The great number of patients undergoing MRE in a single referral center and the observed increasing trend in this number provide a great opportunity for further studies on MRE both as a diagnostic tool and as a monitoring tool for follow-up of the course of the disease in such patients.

\section{CONFLICT OF INTEREST}

The authors declare no conflict of interest related to this work.

\section{REFERENCES}

1. Loftus EV Jr, Schoenfeld P, Sandborn WJ. The epidemiology and natural history of Crohn's disease in population-based patient cohorts from North America: a systematic review. Aliment Pharmacol Ther 2002;16:51-60. doi: j.1365-2036.2002.01140.x

2. Koh DM, Miao Y, Chinn RJ, Amin Z, Zeegen R, Westaby $\mathrm{D}$, et al. MR imaging evaluation of the activity of Crohn's disease. AJR Am J Roentgenol 2001;177:1325-32. doi: 10.2214/ajr.177.6.1771325

3. Low RN, Francis IR, Politoske D, Bennett M. Crohn's disease evaluation: comparison of contrast-enhanced MR imaging and single-phase helical CT scanning. JMagn Reson Imaging 2000;11:127-35. doi: 10.1002/(SICI)15222586(200002)11:2<127::AID-JMRI8>3.0.CO;2-G 
4. Miao YM, Koh DM, Amin Z, Healy JC, Chinn RJ, Zeegen $\mathrm{R}$, et al. Ultrasound and magnetic resonance imaging assessmentof active bowel segments in Crohn's disease. Clin Radiol 2002;57:913-8. doi: 10.1053/crad.2002.1059

5. Magnano G, Granata C, Barabino A, Magnaguagno F, Rossi U, Calevo MG, et al. Polyethylene glycol and contrast-enhanced MRI of Crohn's disease in children: preliminary experience. Pediatr Radiol 2003;33:385-91. doi: $10.1007 / \mathrm{s} 00247-003-0903-\mathrm{z}$

6. Potthast S, Rieber A, Von Tirpitz C, Wruk D, Adler G , Brambs HJ. Ultrasound and magnetic resonance imaging in Crohn's disease: a comparison. Eur Radiol 2002;12:1416-22. doi: 10.1007/s00330-001-1191-3

7. Ajaj WM, Lauenstein TC, Pelster G, Gerken G, Ruehm SG, Debatin JF, et al. Magnetic resonance colonography for the detection of inflammatory diseases of the large bowel: quantifying the inflammatory activity. Gut 2005;54:257-63. doi: 10.1136/gut.2003.037085

8. Eugene C. The second European evidence-based Consensus on the diagnosis and management of Crohn's disease. Clin Res Hepatol Gastroenterol 2011;35:257-9. doi: 10.1016/j.clinre.2011.02.005

9. Florie J, Wasser MN, Arts-Cieslik K, Akkerman EM, Siersema PD, Stoker J. Dynamic contrast-enhanced MRI of the bowel wall for assessment of disease activity in Crohn's disease. AJR Am J Roentgenol 2006;186:138492. doi: 10.2214/AJR.04.1454

10. Aghazadeh R, Zali MR, Bahari A, Amin K, Ghahghaie F , Firouzi F. Inflammatory bowel disease in Iran: a review of 457 cases. J Gastroenterol Hepatol 2005;20:1691-5. doi: 10.1111/j.1440-1746.2005.03905.x

11. Gasparetto M , Guariso G. Crohn's disease and growth deficiency in children and adolescents. World J Gastroenterol 2014;20:13219-33. doi: 10.3748/wjg.v20.i37.13219

12. Wilkens R, Novak KL, Lebeuf-Taylor E, Wilson SR. Impact of intestinal ultrasound on classification and management of Crohn disease patients with inconclusive colonoscopy. Can J Gastroenterol Hepatol 2015.

13. Booya F, Fletcher JG, Huprich JE, Barlow JM, Johnson $\mathrm{CD}$, Fidler JL, et al. Active Crohn disease: CT findings and interobserver agreement for enteric phase CT enterography. Radiology 2006;241:787-95. doi: 10.1148/ radiol.2413051444

14. Raptopoulos V, Schwartz RK, McNicholas MM, Movson J, Pearlman J , Joffe N. Multiplanar helical CT enterography in patients with Crohn's disease. AJR Am J Roentgenol 1997;169:1545-50. doi: 10.2214/ajr.169.6.9393162

15. Jaffe TA, Gaca AM, Delaney S, Yoshizumi TT, Toncheva G, Nguyen G, et al. Radiation doses from small-bowel follow-through and abdominopelvic MDCT in Crohn's disease. AJR Am J Roentgenol 2007;189:1015-22. doi: 10.2214/AJR.07.2427

16. Silverstein J, Grand D, Kawatu D, Shah SA, Steinkeler J, LeLeiko N. Feasibility of using MR enterography for the assessment of terminal ileitis and inflammatory activity in children with Crohn disease. J Pediatr Gastroenterol Nutr 2012;55:173-7. doi: 10.1097/MPG.0b013e318249595d

17. Buchanan GN, Halligan S, Bartram CI, Williams AB, Tarroni D, Cohen CR. Clinical examination, endosonography, and MR imaging in preoperative assessment of fistula in ano: comparison with outcome-based reference standard. Radiology 2004;233:674-81. doi: 10.1148/radiol.2333031724

18. Morris J, Spencer JA, Ambrose NS. MR imaging classification of perianal fistulas and its implications for patient management. Radiographics 2000;20:623-35; discussion 35-7. doi: 10.1148/radiographics.20.3.g00mc15623

19. Baker ME, Einstein DM, Veniero JC. Computed tomography enterography and magnetic resonance enterography: the future of small bowel imaging. Clin Colon Rectal Surg 2008;2:193-212. doi: 10.1055/s-2008-1080999

20. Fidler J. MR imaging of the small bowel. Radiol Clin North Am 2007;45:317-31. doi: 10.1016/j.rcl.2007.03.012

21. Tolan DJ, Greenhalgh R, Zealley IA, Halligan S, Taylor SA. MR enterographic manifestations of small bowel Crohn disease. Radiographics 2010;30:367-84. doi: $10.1148 /$ rg.302095028

22. Lee SS, Kim AY, Yang SK, Chung JW, Kim SY, Park $\mathrm{SH}$, et al. Crohn disease of the small bowel: comparison of CT enterography, MR enterography, and small-bowel follow-through as diagnostic techniques. Radiology 2009;251:751-61. doi: 10.1148/radiol.2513081184

23. Church PC, Turner D, Feldman BM, Walters TD, Greer ML, Amitai MM, et al. Systematic review with metaanalysis: magnetic resonance enterography signs for the detection of inflammation and intestinal damage in Crohn's disease. Aliment Pharmacol Ther 2015;41:15366. doi: 10.1111/apt.13024

24. Punwani S, Rodriguez-Justo M, Bainbridge A, Greenhalgh R, De Vita E, Bloom S, et al. Mural inflammation in Crohn disease: location-matched histologic validation of MR imaging features. Radiology 2009;252:712-20. doi: 10.1148/radiol.2523082167 